\title{
Quality of anticoagulation management with warfarin among outpatients in a tertiary hospital in Addis Ababa, Ethiopia: a retrospective cross-sectional study
}

\author{
Teferi Gedif Fenta ${ }^{1 *}$, Tamrat Assefa ${ }^{1}$ and Bekele Alemayehu ${ }^{2}$
}

\begin{abstract}
Background: Warfarin is the most widely used anticoagulant in the world. The difficulty of managing warfarin contributes to great potential for patient harm, both from excessive anticoagulation and insufficient anticoagulation. This study assessed the International Normalized Ratio (INR) control outcome measures and warfarin dose adjustment practices at cardiology and hematology outpatient clinics at a teaching hospital in Addis Ababa, Ethiopia.

Methods: The study was based on a cross - sectional study design involving 360 retrospective patients' chart review among outpatients who received warfarin for its various indications.

Results: The mean frequency of INR monitoring per patient was 62.9 days (17.2-143.7 days).

Patients spent $52.2 \%, 29.0 \%$ and $18.8 \%$ of the time in sub-therapeutic, therapeutic and supra-therapeutic ranges, respectively. The daily warfarin dose was increased $50.9 \%$ and $36.9 \%$ and decreased in $52.8 \%$ and $60.9 \%$ of the time for occurrences of sub-therapeutic and supra-therapeutic INRs to achieve target ranges of 2.0-3.0 and 2.5-3.5, respectively.

Conclusion: The quality of anticoagulation management with warfarin among outpatients in Tikur Anbessa Specialized Hospital was sub-optimal. This was reflected by low Time in Therapeutic Range (TTR), longer than recommended INR monitoring frequency, and minimal actions taken to adjust warfarin dose after occurrences of non-therapeutic INRs.
\end{abstract}

Keywords: Warfarin, Inr, Time in therapeutic range, Tikur Anbessa specialized hospital

\section{Background}

Since 1954, warfarin has been the most commonly prescribed oral anticoagulant for prevention and treatment of thromboembolic events mainly due to ease of its administration [1]. A fundamental step in quality care research in anticoagulation is establishing that anticoagulation does in fact prevent thromboembolism. During the last 50 years, the efficacy of warfarin in the prevention and treatment of thromboembolic diseases has been firmly established, especially for atrial fibrillation, venous thromboembolism, and valvular heart diseases [2].

\footnotetext{
* Correspondence: tgedif@gmail.com

${ }^{1}$ School of Pharmacy, College of Health Sciences, Addis Ababa University, Addis Ababa, Ethiopia

Full list of author information is available at the end of the article
}

A necessary first step was the development of a standardized test to measure the degree of anticoagulation. The prothrombin time was used to monitor anticoagulation but was plagued by a lack of standardization between laboratories. This was resolved through the development and widespread adoption of INR, which allows results from different laboratories to be comparable [3-5]. Having agreed on a way to measure anticoagulation, investigators were now able to achieve consensus regarding optimal INR ranges for various indications [6].

Despite its efficacy, warfarin is notoriously difficult to manage: its therapeutic window is narrow, it has significant interactions with diet and other medications, and its action is affected by comorbid conditions and other 
inherent patient characteristics. Therefore, it is a major patient safety goal to improve the quality of oral anticoagulation care and there is a need to institute quality measuring programs [7].

A quality measure of anticoagulation management is used to ascertain the degree to which a given system is successfully coordinating care to accomplish a particular therapeutic goal $[7,8]$. Quality of warfarin management is assessed by the proportion of time the patient is maintained within therapeutic range since an increased Time in Therapeutic Range (TTR) is associated with a reduction of hemorrhage and thromboembolism [9]. Quality of warfarin therapy in long-term care was not studied yet in Ethiopian health facilities. Thus, this study was designed to assess the quality of anticoagulation management with warfarin among outpatients in Tikur Anbessa Specialized Hospital (TASH), a large teaching hospital in Addis Ababa. Knowledge of the quality of anticoagulation management with warfarin is important to identify the the most feasible and appropriate intervention to enhance anticoagulation services of TASH.

In the current study we aimed at assessing the INR control measures (proportion/number of INRs within therapeutic range, TTR and standard deviation (SD) of INR values) for warfarin therapy and investigating the quality of warfarin dose adjustment practice (warfarin prescribing) for outpatients in the cardiology and hematology clinics (CHCs) of TASH.

\section{Methods}

\section{The study setting}

The study was carried out at TASH, Addis Ababa, Ethiopia. It is the biggest university teaching hospital in the country and its services are divided as outpatient, inpatient and emergency departments. The hospital has currently about 2000 medical and non-medical staff. It has more than 500 beds [10]. Cardiology and hematology clinics (CHCs) of TASH give health care services for patients who need treatment and prevention of cardiovascular and hematological disorders.

\section{Study design}

The study was based on cross-sectional study design in which retrospective patients' charts review was employed as the data collection technique. The study population was adult patients who had received warfarin for its various indications after visiting the CHCs of TASH.

\section{Sample size determination and patient selection}

The charts of all patients age 18 and above years old and who had received warfarin for at least 3 months and had been followed in CHCs of TASH from January, 2011 to January, 2012 were reviewed. These criteria were set because it is difficult to assess the quality of INR outcome measures in new patients due to instability of INR readings at this stage. Accordingly, 360 patients' charts were included in this study.

Information about demographic and clinical characteristics of patients such as age, sex, comorbid conditions, indications of warfarin therapy, target INR ranges, INR values, frequency of INR monitoring (time interval until next scheduled test) and dose of warfarin patient take per day was gathered using a standardized data abstraction format. After collecting this information from chart, INR control outcome measures: proportion (number) of INRs within therapeutic range, TTR and standard deviation (SD) of INR value [11] were determined and compared with past studies' findings to assess the quality of INR monitoring. The assessment of quality of warfarin prescribing was made based on how non-therapeutic INRs were managed; that is lowering, increasing or omitting dose of warfarin [12]. The time the patients spent in therapeutic range was calculated by the fraction of INR's in the range for different target range of warfarin indications. The following method (formula) was used to determine TTR [9].

\section{TTR $=$ The number of INRs within the target range for all patients Total number of INRs during the selected interval of time}

\section{Data quality assurance and analysis}

Data from chart reviews were based on a standardized data abstraction format to maintain consistency. Data were entered into a computer using Epi Info version 3.5.3 and analyzed by SPSS version 16 and where appropriate, results were expressed as percentages, mean scores, and means \pm standard deviation or ranges.

\section{Results}

The socio-demographic characteristics of outpatients Among 360 outpatients who were attended $\mathrm{CHCs}$ of TASH and received warfarin during the period of January, 2011 to January, 2012; 233 (64.7\%) were females and the mean age was $35.3(\mathrm{SD}=12.8)$ years, with age range from 18 to 85 years. These patients were diagnosed with multiple diseases as co-morbid conditions (Table 1).

\section{The primary indications for warfarin}

As shown in Fig. 1, the primary indications of warfarin therapy were valvular heart diseases (VHD), atrial fibrillation (AF) and mechanical mitral valve replacement (MVR). There were cases where patients received warfarin for more than one indication (48.9\%). The INR target for atrial fibrillation, deep vein thrombosis, pulmonary embolism, valvular heart diseases, bioprosthetic valve replacement in mitral position antiphospholipid antibody syndrome, myocardial infarction, cardiac 
Table 1 Socio-demographic characteristics and comorbidities of outpatients who were on warfarin therapy at $\mathrm{CHCs}$ of TASH, $2012(N=360)$

\begin{tabular}{lll}
\hline Item Description & & $\mathrm{N}(\%)$ \\
\hline Sex & Male & $127(35.3)$ \\
& Female & $233(64.7)$ \\
Age & $18-30$ & $155(43.1)$ \\
& $31-45$ & $140(38.9)$ \\
& $46-60$ & $42(11.7)$ \\
& Above 60 & $23(6.3)$ \\
Comorbid conditions & Hypertension & $37(10.3)$ \\
& Congestive Heart Failure & $154(42.8)$ \\
& Asthma & $6(1.7)$ \\
& Hyper/Hypothyroidism & $3(0.8)$ \\
& Pulmonary Hypertension & $62(17.2)$ \\
& Others ${ }^{a}$ & $60(16.7)$
\end{tabular}

${ }^{a}$ Others include anemia, osteoarthritis, neurologic disorders, depression, peptic ulcer disease, gasteroesophageal reflux disease, rheumatoid arthritis, infectious diseases, renal disorders, HIV/AIDS, ischemic heart disease, hemiparesis, congenital heart disease, cancer, etc

embolic stroke, cardiomyopathy, dilated cardiomyopathy and peripheral vascular disease was $2-3$. Whereas INR target of 2.5-3.5 was used for mechanical valve replacement in mitral position, and for dual aortic and mitral mechanical valve replacement.

\section{INR distribution and outcome measures}

A total of 2245 INRs values were recorded over 12 months. They were divided into different categories to show how INRs were distributed. As indicated in Fig. 2, 484(21.6\%) and $527(23.5 \%)$ of the INRs values were $\leq 1.5$ and in the range of $>1.5$ to 2 , respectively. Out of all INR values, $138(6.1 \%)$ were above 5 . The mean frequency of INR monitoring per patient was every62.9 days (range
17.7-143.7 days) irrespective of patients' INR targets. Furthermore, average follow up time for INR values was calculated for both targets with their respective non-therapeutic INR monitoring frequency as described in Fig. 3. INR goal was $2.0-3.0$ in $66.7 \%$ and $2.5-3.5$ in $33.3 \%$ of patients. The mean INR reading per patient was $2.5 \pm 0.8$ and $2.6 \pm 0.80$ in those who should achieve INR of 2.0-3.0 and 2.5-3.5, respectively. The time patients spent in the sub-therapeutic, within therapeutic and supra-therapeutic ranges was shown in Fig. 4 for both INR rages. On average patients spent $52.2 \%, 29 \%$ and $18.8 \%$ of the time in subtherapeutic, therapeutic and supra-therapeutic ranges, respectively.

\section{Warfarin dose adjustment practices at TASH}

The assessment of quality of warfarin prescribing (daily warfarin dose adjustment practice) was made based on how non-therapeutic INRs were managed; that is lowering, increasing or omitting dose of warfarin [12]. In the target range of 2.0-3.0, for INRs that were $<2.0$, the average daily dose of warfarin for the days that followed was increased in $276(50.9 \%)$ of such cases. In 190 (35.1\%) of such cases, physicians did not change the previous dose of warfarin. However, for 76 (14.2\%) of subtherapeutic INRs of target range 2.0-3.0, the average daily dose of warfarin was decreased until next patient appointment date. Among 320 INRs which were above target range $(>3.0)$, warfarin daily dose was decreased in $169(52.8 \%)$ of occasions. But in 106 (33.1\%) and 45 (14.1\%) of occurrences (INRs > 3), warfarin daily dose was remained unchanged and increased for the days that followed, respectively (Table 2).

For patients who were supposed to achieve a target range 2 (2.5 to 3.5$)$, but were in sub-therapeutic range $($ INR $<2.5)$, physicians increased daily warfarin dose in

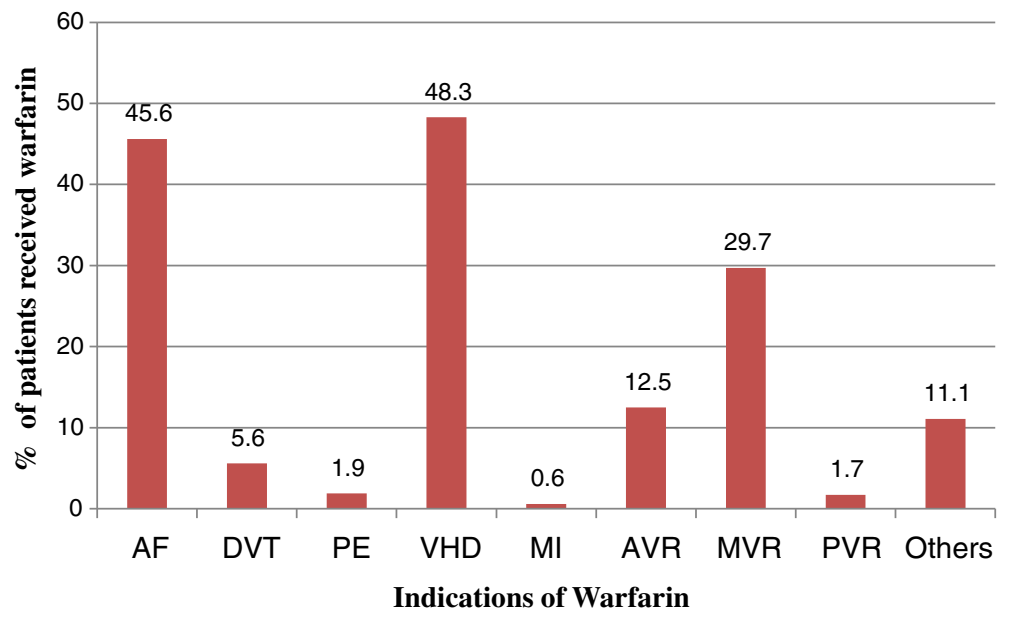

Fig. 1 Indications of warfarin therapy for outpatients in CHUs of TASH, 2012 


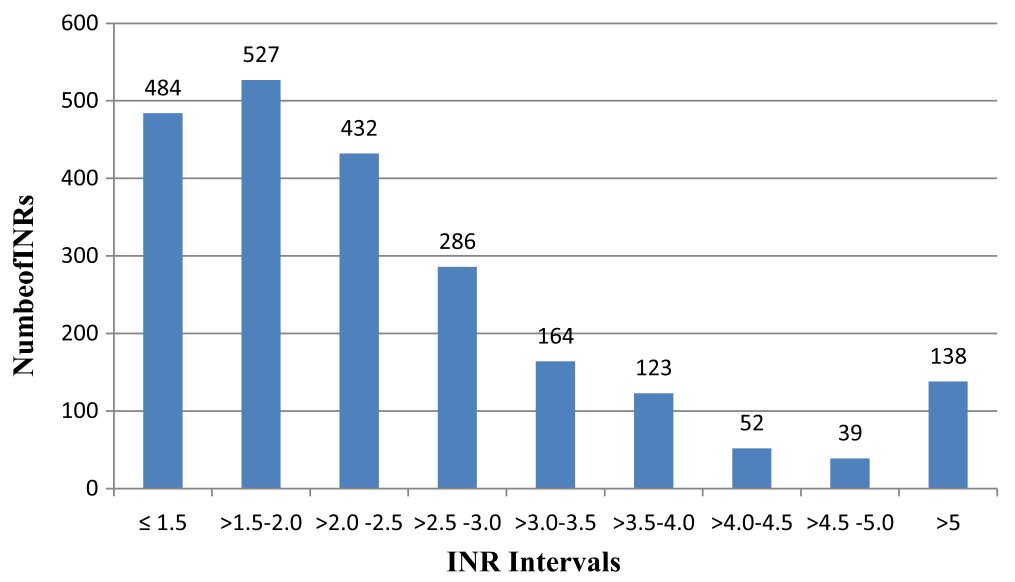

Fig. 2 INR values distributions within different intervals for outpatients who were on warfarin at TASH, 2012

$122(36.9 \%)$, decreased in 56 (16.9\%), and remained unchanged in $153(46.2 \%)$ of cases. For the total of 81 INRs that were above the goal range (INR > 3.5), warfarin daily dose was decreased in $49(60.5 \%)$ of the cases till the patients' next visit.

\section{Discussion}

This study explored the quality of anticoagulation management with warfarin in the CHCs of TASH. Quality of INR monitoring by physicians was unsatisfactory; as revealed by low TTR (29\%) when compared with a goal of maintaining a therapeutic INR $\geq 50 \%$ of the time (the minimum threshold required to achieve a benefit from warfarin therapy) [12]. The potential reasons for low TTR might be prolonged duration of INR monitoring, longer appointment date due to patient load, absensce of a standard protocol for warfarin therapy management and lack of a anticoagulation clinic at the studied hospital.

The TTR obtained in the present study was also lower than the results documented in other studies [13-15]. A research done in the city of St. John's, Canada among patients at family medicine clinic reported TTR of $65 \%$, just over twice the present study result [16]. However, our result was similar to findings from a study in US hospital which reported $30.6 \%$ TTR [17], but higher than the result of a study done in India [18]. The low TTR might be due to less frequent monitoring (1INR within 63 days). According to one previous study, TTR can be increased with more frequent INR monitoring [18]. It has been also demonstrated that $50-60 \%$ of patients can be expected to remain in their target range if monitoring of INR occurs monthly, 77-85\% if monitored weekly and up to $92 \%$ if monitored every 3 days [19]. Moreover, the present study

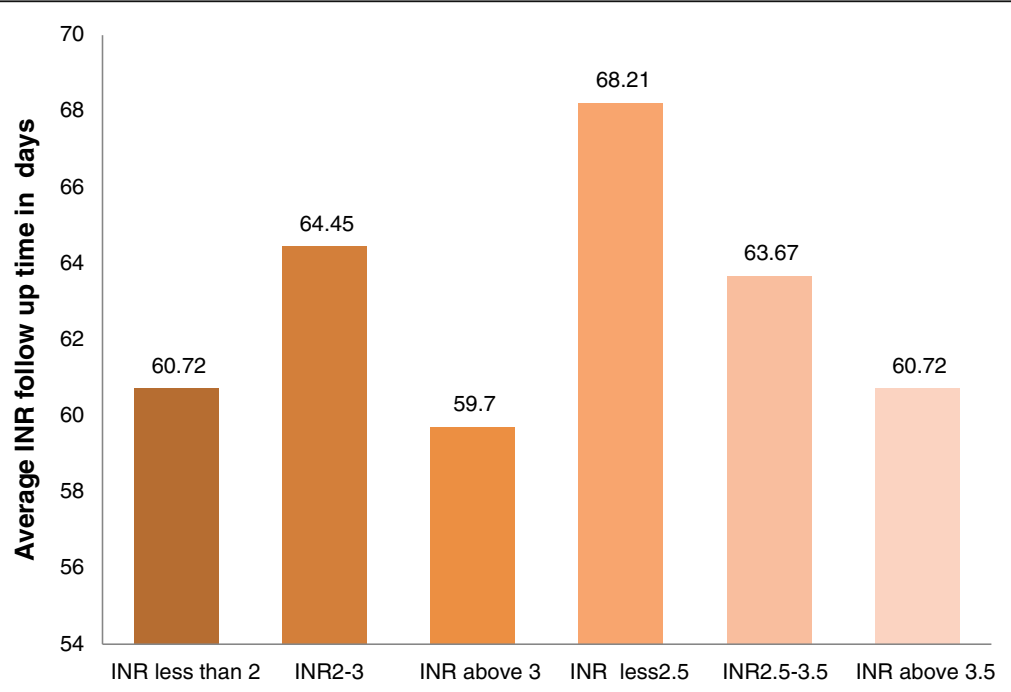

Fig. 3 Average INR follow up time in days for different INR levels among outpatients who were on warfarin at TASH, 2012 


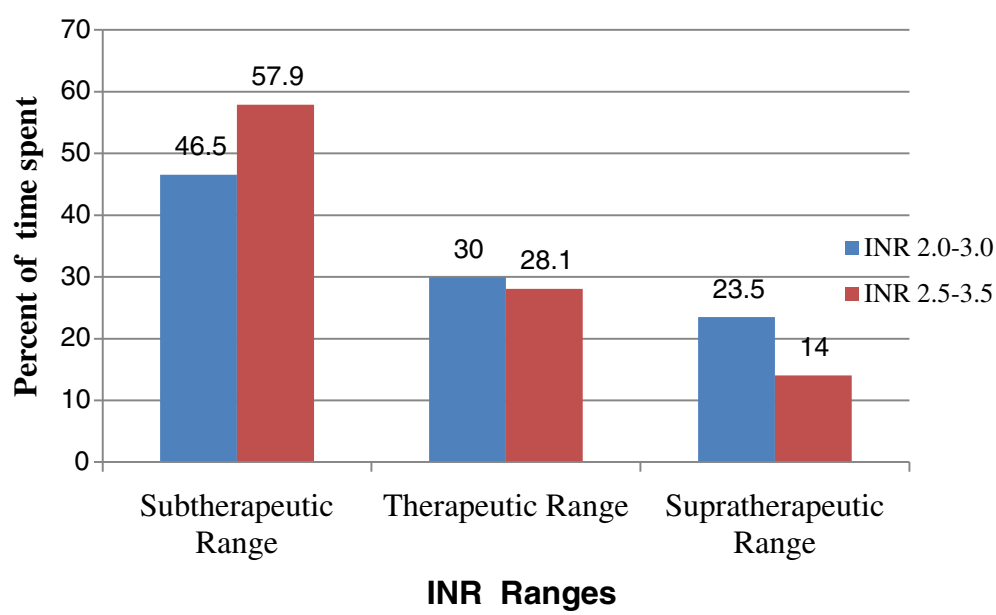

Fig. 4 Time spent in sub-therapeutic, therapeutic and supra-therapeutic INR ranges for outpatients who were on warfarin at TASH, 2012

revealed that patients spent $18.8 \%$ of the time in supra-therapeutic range. This figure was higher than results previously reported (11-14\%) [12, 13, 15, 20].

The mean interval between two INR tests per patient was nearly 63 days among follow up patients in the $\mathrm{CHCs}$ of TASH which was very long even for patients who were receiving a stable dose warfarin. A study done in United State of America showed INR was monitored every 46 days [17]. Whereas, another study found that the mean number of days between two INRs determinations was 6.2 [11]. The longer INR monitoring frequency could be one of the main reasons for the lower TTR identified in this study. The America College of Chest Physicians and American Heart Association and other studies suggest that INR should be monitored at an interval of no longer than every 4 weeks for stable patients receiving warfarin $[5,8,21]$.

Management of non-therapeutic INRs (quality of warfarin prescribing) by physicians was sub-optimal. In this regard, it was in only $276(50.9 \%)$ and $122(36.9 \%)$ of cases in which daily doses of warfarin increased for the days followed in response to occurrences of subtherapeutic ranges in relation to target ranges of 2.0-3.0 and 2.5-3.5, respectively. The finding of the present study was very small when compared with what was found by Aspinall et al., [12] where in 75\% of cases warfarin doses were increased following occurrences of sub- therapeutic ranges. Moreover, the present study indicated that warfarin dose adjustment at TASH was also minimal when INRs were in supra-therapeutic ranges. This was reflected by decreased daily dose of warfarin in only $169(52.8 \%)$ and $49(60.5 \%)$ of the cases in the two target INR ranges.

INR should be monitored within a week after the occurrences of non-therapeutic ranges to adjust daily warfarin dose accordingly [8]. Taking other alternative actions such as recommending non- pharmacological actions and managing warfarin interacting medications could be the reasons for providers' poor response on warfarin dose adjustment for patients in non-therapeutic INR ranges $[8,22,23]$. However, our study didn't assess the influence of the above alternative interventions due to poor patients' medical charts recording practice in the units and high turnover of physicians in managing the same patient.

The anticoagulation services are being provided once in a week in TASH. This would influence the management of non-therapeutic INRs and warfarin dose adjustment. For managing the occurrence of INR readings out of the therapeutic ranges, both units should use warfarin dosing algorithms. High quality dose management is needed to achieve and maintain the INRs in the therapeutic ranges. Paper-based and/or computerized software models of warfarin-dosing protocols, use of point

Table 2 Non-therapeutic INRs monitoring and daily warfarin dose adjustment in the CHUs of TASH, 2012

\begin{tabular}{lllll}
\hline Target INR Range & Number of Non-therapeutic INRs & Decreased N (\%) & Unchanged N (\%) & Increased N (\%) \\
\hline Target Range 1 & INR $<2(N=542)$ & $76(14.2)$ & $190(35.1)$ & $106(33.1)$ \\
Target Range 2 & INR $>3(N=320)$ & $169(52.8)$ & $150.9)$ & $45(14.1)$ \\
& INR $<2.5(N=331)$ & $56(16.9)$ & $153(46.2)$ & $122(36.9)$ \\
& INR $>3.5(N=81)$ & $49(60.5)$ & $17(21)$ & $15(18.5)$ \\
\hline
\end{tabular}

Target Range $1=$ INR 2.0-3.0 Target Range $2=$ INR 2.5-3.5 
of care testing, anticoagulation management services with dedicated personnel (i.e. anticoagulation clinics) are recommended tools to provide a systematic approach to improve anticoagulation management services [17].

In settings like the $\mathrm{CHCs}$ of TASH where physicians are over burdenend with diagnosing and treating all patients' disease conditions and a shortage of physicians is evident, clinical pharmacist can provide intense education to patients and their caregivers, check patients' adherence more thoroughly, give extra attention to potential warfarin- drug/herbal supplement interaction at each clinic visit and can counsel patients who have poor adherence to their treatment and difficulty in INR control [24]. Pharmacist managed anticoagulation clinics are becoming common in hospitals of most countries and have shown improved patient outcomes including reduced hospital admissions for preventable embolism, bleeding, or treatment of thrombosis [16, 25-27]. For example, implementation of a pharmacist managed ambulatory care anticoagulation clinic in South Korea resulted in an increase in percentage of INRs maintained within therapeutic range from $66 \%$ in the usual care group to $82 \%$ with the new initiative [28]. Thus, based on finding from this study and internationational experiences, there are opportunities to start Pharmacist Managed Anticougulation Clinic in this biggest teaching hospital (TASH) in the country.

The present study is not without limitations. While auditing patients' charts retrospectively, we faced a continuous challenge to extract the necessary information. This was mainly due to poor organization in documenting of patients' history chronologically and; also illegible physician handwriting. Beside this, while searching charts of the study population from the hospital medical record room, we couldn't find a large number of charts which might have important information due to poor documentation and arrangement. Moreover, the incompleteness of the patient charts hindered the ability to identify the number of bleeding or thromboembolic events from the study population.

\section{Conclusions}

From this study it can be concluded that the quality of anticoagulation management with warfarin among outpatients who received warfarin was suboptimal. This was reflected by low TTR, longer INR monitoring frequency than is normally recommended, and minimal actions taken by physician to adjust daily warfarin dose after occurrence of non-therapeutic INRs.

\section{Abbreviations}

CHCs: Cardiac and Hematology Clinics; INR: International Normalized Ratio; PMAC: Pharmacist Managed Anticougualtion Clinic; SD: Standard Deviation; TASH: Tikur Anbessa Specialized Hospital; TTR: Time in Therapeutic Range

\section{Acknowledgements}

The authors would like to thank the Graduate Programs of Addis Ababa University for financial support to do this research and TASH for allowing for collection of information.

\section{Funding}

The research was funded by the Graduate Programs of Addis Ababa University.

\section{Availability of data and materials}

The data used to write this paper are available in the possession of the first author but due to ethical requirements, the raw data cannot be shared.

\section{Authors' contributions}

TA and TGF conceived the research idea. TA developed the proposal which was enriched and approved by TGF and BA. Then TA collected and analyzed the data and drafted the manuscript. TG and BA did critical revisions of the manuscript for its important intellectual content. All authors read and approved the final manuscript.

\section{Competing interests}

The authors declare that they have no competing interests.

Consent for publication

Not applicable.

\section{Ethics approval and consent to participate}

Ethical approval and permission was obtained from the Department of Pharmacology and Clinical Pharmacy at the School of Pharmacy, College of Health Sciences, Addis Ababa University (AAU). Since the data was taken as part of routine services, participants' consent was not a requirement. Instead, Institutional consent was secured from the Department of Internal Medicine, School of Medicine, AAU, to use the data.

\section{Publisher's Note}

Springer Nature remains neutral with regard to jurisdictional claims in published maps and institutional affiliations.

\section{Author details}

${ }^{1}$ School of Pharmacy, College of Health Sciences, Addis Ababa University, Addis Ababa, Ethiopia. ${ }^{2}$ School of Medicine, College of Health Sciences, Addis Ababa University, Addis Ababa, Ethiopia.

Received: 17 September 2015 Accepted: 22 May 2017

Published online: 06 June 2017

\section{References}

1. Hasan SS, Shamala R, Syed IA, Basarian N, Chong DWK, Mei TK, et al. Factors affecting Warfarin-related Knowledge and INR control of patients attending physician- and pharmacist-managed anticoagulation clinics. J Pharm Pract. 2011;24:485-93.

2. Pirmohamed M. Warfarin: almost 60 years old and still causing problems. Br J Clin Pharmacol. 2006;62:509-11.

3. Ageno W, Gallus AS, Wittkowsky A, Crowther M, Hylek EM, Palareti G. Oral anticoagulant therapy: antithrombotic therapy and prevention of thrombosis. Chest. 2012;141:e44S-88S.

4. Boroumand M, Goodarzynejad H. Monitoring of anticoagulant therapy in heart disease: considerations for the current assays. Teh Univ Heart Ctr. 2010;2:57-68.

5. Hirsh J, Fuster V, Ansell J, Halperin JL. American Heart Association/American College of Cardiology Foundation guide to Warfarin therapy. Circulation. 2003;107:1692-711.

6. Gogna A, Arun S. Anticoagulation in clinical practice. Jiacm. 2005;6:53-66.

7. Rose AJ, Berlowitz DR, Frayne SM, Hylek EM. Measuring quality of oral anticoagulation care: extending quality measurement to a new field. Jt Comm J Qual Patient Saf. 2009;35:146-55.

8. Holbrook A, Schulman S, Witt DM, Vandvik PO, Fish J, Kovacs MJ, et al. Evidence-based Management of Anticoagulant Therapy: antithrombotic 9th ed: American College of Ches Physicians Evidence -based clinical practice guidelines. Chest. 2012;141:e152S-84S. 
9. Schmitt L. Speckman J and Ansell. Quality assessment of anticoagulation dose management: comparative evaluation of measures of time in therapeutic range. J Thromb Thrombolysis. 2003;15:213-6.

10. Drug and Therapeutics Committee of TASH. List of Pharmaceuticals for Tikur Anbessa Specialized Hospital. 1st ed, Addis Ababa: TASH; 2012.

11. Baker JW, Pierce KL, Ryals CA. INR goal attainment and oral anticoagulation Knowledge of patients enrolled in an anticoagulation Clinic in a Veterans Affairs Medical Center. J Manag Care Pharm. 2011;17:133-42.

12. Aspinall SL, Zhao X, Handler SM, Stone RA, Kosmoski JC, Libby EA, et al. The quality of Warfarin prescribing and monitoring in veterans affairs nursing homes. Am Geriatr soc. 2010:58:1475-80.

13. McCormick D, Gurwitz JH, Goldberg RJ, Becker R, Tate JP, Elwell A, et al. Prevalence and quality of Warfarin use for patients with Arial fibrillation in the long-term care setting. Arch Intern med. 2001;161:2458-63.

14. Shin $\mathrm{H}$, Kim Y, Bae H, Lee H, Cho H, Cho Y. Trends in oral anticoagulation therapy among Korean patients with Atrial fibrillation: the KORean Atrial fibrillation investigation. Korean Circ J. 2012:42:113-7.

15. Verhovsek M, Motlagh B, Crowther MA, Kennedy C, Dolovich L, Campbell G, et al. Quality of anticoagulation and use of warfarin-interacting medications in long-term care: a chart review. BMC Geriatr. 2008;8:13.

16. Young S, Bishop L, Twells L, Dillon C, Hawboldt J, O'Shea P. Comparison of pharmacist managed anticoagulation with usual medical care in a family medicine clinic. BMC Fam Pract. 2011;12:88.

17. Franke CA, Dickerson LM, Carek PJ. Improving anticoagulation therapy using point-of-care testing and a standardized protocol. Ann Fam med. 2008;6: 28-32.

18. Kakkar N, Kaur R. Knowledge Base of clinicians regarding oral anticoagulant therapy in a teaching - a questionnaire survey JAPI. J Assoc Physicians India. 2004;52:868-87.

19. Khan TI, Kamali F, Kesteven P, Avery P, Wynne H. The value of education and self- monitoring in the management of warfarin therapy in older patients with unstable control of anticoagulation. $\mathrm{Br} J$ Haematolo. 2009;126: 557-64.

20. Papaioannou A, Kennedy CC, Campbell G, Stroud JB, Wang L, Dolovich L. A team-based approach to warfarin management in long term care: a feasibility study of the MEDeINR electronic decision support system. BMC Geriatr. 2010:10:38

21. Dolan G, Smith LA, Collins JM, Plumb JM. Effect of setting, monitoring intensity and patient experience on anticoagulation control: a systematic review and meta analysis of th literature. Curr med res Opin. 2008;24:1459-72.

22. Phillips KW, Ansell J. Outpatient management of oral vitamin K antagonist therapy: defining measuring high-quality management. Expert Rev Cardiovas Ther. 2008;6:57-70.

23. Keeling D, Baglin T, Tait C, Watson H, et al. Guidelines on oral anticoagulation with warfarin - fourth edition. Br J Haematolo. 2011;254:311-24.

24. Chan FWH, Wong RSM, Lau WH, Chan TYK, Cheng G, You JHS. Management of Chinese patients on warfarin therapy in two models of anticoagulation service - a prospective andomized trial. Br J Clin Pharmacol. 2006;62:601-9.

25. Poon IO, Lal L, Brown EN, Braun UK. The impact of pharmacist-managed anticoagulation therapy in older veterans. J Clin Pharm Ther. 2007;32:21-9.

26. Bungard TJ, Grardner L, Archer SL, Hamilton P, Ritchie B, Tymchak W, et al. Evaluation of a pharmacist-managed anticoagulation clinic: improving patient care. Open Med. 2009;3:e16-21.

27. Garton L, Crosby JF. A retrospective assessment comparing pharmacist managed anticoagulation clinic with physician management using international normalized ratio stability. J Thromb Thrombolysis. 2011;32:426. doi:10.1007/s11239-011-0612-7.

28. Choe HM, Kim J, Choi KE, Mueller BA. Implementation of the first pharmacist managed ambulatory care anticoagulation clinic in South Korea. Am J Health-Syst Pharm. 2002:59:872-4.

\section{Submit your next manuscript to BioMed Central and we will help you at every step:}

- We accept pre-submission inquiries

- Our selector tool helps you to find the most relevant journal

- We provide round the clock customer support

- Convenient online submission

- Thorough peer review

- Inclusion in PubMed and all major indexing services

- Maximum visibility for your research

Submit your manuscript at www.biomedcentral.com/submit
Biomed Central 